\title{
Functional magnetic resonance imaging of source versus item memory
}

\author{
Jin Fan, ${ }^{\mathrm{CA}}$ Joan Gay Snodgrass' and Robert M. Bilder²
}

\begin{abstract}
Sackler Institute for Developmental Psychobiology, Department of Psychiatry, Weill Medical College of Cornell University, I300 York Avenue, Box 140, New York, NY 10021; 'Department of Psychology, New York University; ${ }^{2}$ Center for Advanced Brain Imaging, Nathan S. Kline Institute for Psychiatric Research, USA

${ }^{\mathrm{CA} C o r r e s p o n d i n g ~ A u t h o r: ~ j i f 2004 @ m e d . c o r n e l l . e d u ~}$

Received 2 June 2003; accepted 9 June 2003
\end{abstract}

DOI: 10.1097/0I.wnr.0000090582.35425.el

Both the frontal lobes and the medial temporal lobe (particularly the hippocampus) have been implicated in encoding and retrieval of episodic memory information. We report an experiment that manipulates whether source information, item information, or both are required at retrieval. Two sources were used in a factorial design in which the main effect of source and item retrieval, along with their interaction, could be measured by fMRI activations.
When source information was required at retrieval the left frontal lobe showed significant activation but not when item retrieval was required. Hippocampal activation showed no difference between source and item retrieval. This pattern of results supports a model proposing a larger role for the frontal lobes in encoding and retrieval of source information. NeuroReport 14:2275-2281 (c) 2003 Lippincott Williams \& Wilkins.

Key words: fMRI; Item memory; Source memory

\section{INTRODUCTION}

Episodic memory is memory for events that occurred in a particular place at a particular time [1]. Episodic memory contains information about the content of an experience (item), as well as its context (source) [2]. Some researchers have argued for a dual-process model of source and item memory whereas others have argued for a single process model, in which source memory does not differ in kind from item memory but simply reflects a more elaborated memory trace.

Dual-process model: The dual-process model of source vs item memory for the brain holds that the prefrontal regions subserve source memory and the hippocampal regions subserve item memory [3]. Evidence for the dual-process model initially came from studies of patients with frontal lobe dysfunction, who often show normal item memory but deficient source memory. For example, patients with frontal damage who recall or recognize information at normal levels may show disrupted memory for temporal order [4]. While lesion evidence suggests that memory for source is subserved by the frontal lobes [5,6], similar clinical data suggest that the recognition of an item is subserved by medial temporal and/or diencephalic structures [7], supported by a double dissociation between item memory and source memory in elderly subjects [8]. A PET study has supported the dual brain process model [9]. It is found that item retrieval is related to increased activity in medial temporal and basal forebrain regions, whereas temporal order (source) retrieval is related to activations in dorsal prefrontal, cuneus/precuneus, and right posterior parietal regions.

Single-process model: The single-process model proposes that both frontal and hippocampal areas are more involved in source than in item memory retrieval because retrieval of source information is more difficult than retrieval of item information. Some support for the single process model is provided by studies demonstrating that in addition to the frontal lobes, the medial temporal system (hippocampus and adjacent medial temporal lobe) is important for encoding and retrieving source information [10]. Contradicting the dual-process model, source accuracy was related to scores on the medial temporal measure suggesting that medial temporal structures are involved in the binding of contextual features (source information) to item information [11].

In an event-related fMRI study, prefrontal activity was related more to source than to item retrieval [12]. In this study, four participants performed acquisition tasks with picture and word stimuli by rating the difficulty of drawing each item or by rating the number of possible uses for each item. During a subsequent surprise memory test, participants performed an old/new recognition test or a source (picture $v s$ word) test. The left prefrontal region was more activated in the source than the item test, suggesting that the 
left prefrontal cortex is recruited for more demanding, systematic processes while the right prefrontal cortex subserves relatively simple, heuristic cognitive processes. Another fMRI study manipulating encoding at study found that when retrieval demanded greatest effort due to shallow encoding, the left dorsal prefrontal region was more active during episodic memory retrieval [13].

The studies reviewed above do not provide direct support for either the single-process model or the dual-process model. Such support would require evidence for the role of the hippocampus in source memory and item memory. The present study was designed to distinguish between the dual- and single-process brain models of source and item retrieval. The dual-process model proposes that the prefrontal lobe is involved more in source memory retrieval whereas the hippocampus is involved more in item memory retrieval. This model predicts that the source memory task will produce increased activation in frontal areas, while the item memory task will produce increased activation in the hippocampus. The single-process model proposes that both prefrontal and hippocampal regions are involved in both item and source memory retrieval but that the intensity of activation of both regions will be greater for source than for item memory retrieval.

\section{SUBJECTS AND METHODS}

Participants: Eight right-handed participants between the ages of 18 and 27 (mean age 20.5 years) served in the experiment. Four were assigned to the color source condition and four to the voice source condition. The mean ages were the same for both sets of subjects and each set consisted of two male and two female participants. All participants were right-handed, had normal or corrected-tonormal visual acuity, and normal color vision. No participant reported any left-handed or ambidextral first-degree relatives. Participants provided written informed consent following procedures approved by the Nathan S. Kline Institute for Psychiatric Research and New York University prior to participating.

Apparatus and materials: Pictures and their names were selected from a set of 400 line drawings [14]. The total number of pictures was 384. Pictures were drawn in red, green, or black on a white background on a video monitor connected to a PC. A single female and male recorded the names of each of the pictures. The spoken names were played through the speaker of the MRI scanner and began simultaneously with the presentation of each picture.

Experimental design: Friston and colleagues proposed that factorial designs are more powerful than subtractive designs in characterizing brain cognitive processes because they allow for interactions and avoid assumptions like pure insertion [15]. In a factorial design, the effect of a cognitive component is best captured by the main effect of that component and the integration among components can be assessed with the interaction terms.

Accordingly, this experiment had a factorial design in which both sources (color of picture and gender of voice naming the picture) were manipulated, and in which at test participants were asked to make item judgments, source judgments, both, or neither. The source (color vs voice) was a between-subjects factor. The other factors were withinsubjects factors. Each participant received four conditions (see below) as a run and a total of five runs was presented. There were two phases for each task, the study phase and the test phase. Brain images were acquired during both phases. Items were counterbalanced across conditions and across participants in order to control for item effects.

Cognitive tasks: There were four test conditions. Each test condition was preceded by a study condition. The four test conditions differed in the information asked of the participant. All test conditions were preceded by the same study task in which participants were presented with a series of 16 pictures drawn in red or green (for the color group), or drawn in black accompanied with its name spoken in a female or a male voice (for the voice group). The task of the participants during the study phase was to indicate with a button press whether the picture was red/ green, or the voice was female/male.

In the test phase, the baseline condition (task A, see below) included the perceptual and response requirements without the memory decisions. Successive conditions added either item recognition (task B), source retrieval (task C), or both item recognition and source retrieval (task D). Table 1 presents the four tasks and the behavioral results.

In task A (repetition priming, old items) participants were shown the same items as at study in a different random order and were to make the same responses as at study. Their task was to indicate with a button press whether the picture was red/green, or the voice was female/male. In task B (item recognition only, old/new items) participants were shown half of the study items (eight) mixed with eight new items and were to judge whether the item was old (studied) or new by pressing one key for old and a second key for new. The color group was shown the old pictures drawn in their original colors and the new pictures drawn in either red or green. The voice group was shown the old pictures accompanied by their original voice and the new pictures accompanied by either a male or female voice. In task C (source judgments only, old items) participants were shown only the 16 study items (i.e. all items were old) presented as black on white line drawings and were asked to judge whether the item was shown in red or green during study (color group) or was named by a female or male voice during study (voice group) by pressing one key for source 1 and a second key for source 2 . In task D (item recognition + source judgment, old/new items) participants were shown both studied and new items (as in Task B) presented

Table I. Values of response accuracy and reaction times (ms) for four tasks.

\begin{tabular}{llc}
\hline Item retrieval & \multicolumn{2}{c}{ Source retrieval } \\
\cline { 2 - 3 } & \multicolumn{1}{c}{ Absent } & Present \\
\hline Absent & A $^{\mathrm{a}} 0.99(1258)$ & $\mathrm{C} 0.8 \mathrm{I}(\mathrm{I422})$ \\
Present & $\mathrm{B} 0.88(1447)$ & $\mathrm{D} 0.79(1557)$ \\
\hline
\end{tabular}

${ }^{a}$ Task A: repetition priming (old items); task B: item recognition only (old/ new items); task C: source judgments only (old items); and task D: item recognition + source judgment (old/new items). 
as black on white line drawings and were to make one of three judgments: old (source 1), old (source 2) or new by pressing one of three keys.

With this factorial design, it is possible to separately estimate the activation for item recognition and for source memory retrieval. For the factorial analysis $(B+D)-(A+C)=$ main effect of item recognition, $(C+D)-(A+B)=$ main effect of source retrieval, and $(\mathrm{D}-\mathrm{C})-(\mathrm{B}-\mathrm{A})=$ interaction of item recognition and source retrieval.

Procedures: For each participant, five runs were acquired. Each run consisted of four blocks of task conditions A, B, C, and D. Each block was $128 \mathrm{~s}$ long with 32 trials (16 study trials and 16 test trials). The first run was for practice and was run outside the scanner. The behavioral data from this run were not analyzed. Each run was $8 \mathrm{~min} 32 \mathrm{~s}$ long. The whole experiment took $\sim 40 \mathrm{~min}$. The order of the four test tasks was counterbalanced across participants. Pictures were presented to participants every $4 \mathrm{~s}$ during both study and test. The button-press responses were recorded by the computer.

MR imaging was carried out using a $1.5 \mathrm{~T}$ Siemens Vision scanner. Functional scans were collected using a T2* weighted gradient echo planar imaging (EPI) sequence $\left(\mathrm{TR}=4 \mathrm{~s}, \mathrm{TE}=50 \mathrm{~ms}\right.$, flip angle $\left.=90^{\circ}\right)$ with an in-plane resolution of $3.44 \times 3.44 \mathrm{~mm}(64 \times 64$ matrix; $220 \mathrm{~mm}$ field of view). Twenty-one $5 \mathrm{~mm}$ slices (skip $1 \mathrm{~mm}$ between slices) were acquired along the AC-PC plane as determined on a midsagittal pilot scan. A high resolution T1-weighted structural MRI sequence $(\mathrm{TR}=700 \mathrm{~ms}, \mathrm{TE}=14 \mathrm{~ms}$, flip angle $=70^{\circ}, \mathrm{FOV}=220 \mathrm{~mm}, 256 \times 256$ matrix) was acquired with the same orientation and slice positions and thickness as the functional runs to provide detailed anatomic images aligned to the functional scans.

Data analysis of the fMRI study: Data were analyzed using statistical parametric mapping (SPM99b, Wellcome Department of Cognitive Neurology, London, United Kingdom). All volumes from each participant were realigned to the first volume, co-registered with the participant's T1 structural MRI, normalized to a standard EPI template, and smoothed using an $8 \mathrm{~mm}$ full-width-at-half-maximum (FWHM) Gaussian kernel. After normalization, voxels were resampled with $2 \times 2 \times 2 \mathrm{~mm}^{3}$ voxel size. The data from each participant were analyzed by modeling experimental phases as delayed box-car functions and experimental conditions as contrasts in the context of the general linear model. Images of each condition for each subject were collapsed into a single image. The main effects of item memory, source memory, and their interaction were analyzed. The activated regions were defined as those exceeding both an uncorrected height threshold $(p<0.001)$ and an uncorrected extent threshold $p<0.001 \quad(\mathrm{k}=216$ voxels; $p<0.05$, corrected).

Because hippocampal regions are small with respect to these criteria for judging significant activation based on whole brain volume, a separate region of interest (ROI) analysis was used to test hippocampal activation for four hippocampal regions: left anterior hippocampus $(x=-24$, $\mathrm{y}=-16, \mathrm{z}=-14)$, right anterior hippocampus $(24,-16,-14)$, left posterior hippocampus $(-30,-33,-3)$, and right posterior hippocampus $(30,-33,-3)$. One voxel $\left(2 \times 2 \times 2 \mathrm{~mm}^{3}\right)$ was selected from each region for this analysis. The four voxel coordinates were selected based upon previous results showing functional segregation of the anterior and posterior hippocampus during learning and the particular locations within each region of maximal activation [16-18]. Signal differences (changes) between experimental conditions were tested for the four voxels located at the four pre-defined coordinates.

Using the event-related procedure, we were able to approximate the relationship between evoked neuronal activity and the hemodynamic response by a linear convolution using a fixed and time-invariant hemodynamic response function (hrf). Time series were adjusted for confounds of head motion, low-frequency shifting, global mean, and other components. A low-pass filter (Gaussian $\mathrm{FWHM}=4 \mathrm{~s}$ ) and a high-pass filter for each run (cutoff period $=32 \mathrm{~s}$ ) were used. The scaling method was used for global normalization.

\section{RESULTS}

Because of the similarities in results between the two source conditions, their data were combined for both the behavioral and fMRI analyses.

Behavioral results: The mean accuracy and reaction time for the study phase were 0.99 and $1310 \mathrm{~ms}$, respectively. Table 1 shows the results of the behavioral measurement (accuracy and reaction time) for the four test tasks combined across source conditions. Reaction times have been combined across the four categories of hits, false alarms, correct rejections, and misses. Our major interest in these behavioral results is to ensure that the tasks are ranked in difficulty as they should be (i.e., task A should be the easiest, then $B$ and $C$, with task $D$ being the most difficult).

A 2 (source retrieval: absent, present) $\times 2$ (item retrieval: absent, present) within-subject ANOVA on response accuracy indicated that the main effect of source retrieval was significant $(\mathrm{F}(1,7)=12.13, p<0.01)$. Performance requiring source retrieval was worse than performance not requiring source retrieval. The main effect of item retrieval was marginally significant $(\mathrm{F}(1,7)=5.33, p=0.05)$. Performance requiring item retrieval was worse than performance not requiring item retrieval. There was a significant interaction of item recognition and source retrieval $(\mathrm{F}(1,7)=23.16$, $p<0.01)$. The interaction is produced by the lack of difficulty of the baseline task A (both item and source retrieval absent).

A 2 (source retrieval: absent, present) $\times 2$ (item retrieval: absent, present) within-subject ANOVA on reaction time indicated that the main effect of source retrieval showed a non-significant trend $(\mathrm{F}(1,7)=4.71, p=0.07)$. The mean reaction time for source retrieval tended to be longer than the mean reaction time for conditions that did not require source retrieval. The main effect of item retrieval was significant $(\mathrm{F}(1,7)=6.23, p<0.05)$. The mean reaction time for item retrieval was longer than the mean reaction time for conditions that did not require item retrieval. The interaction of item recognition and source retrieval was not significant $(\mathrm{F}<1)$. 
fMRI results: The fMRI results of the color and voice conditions showed that for both sources, left frontal regions showed activation for source memory retrieval, although they were not in the same Brodmann area (BA 10, $-32,55,5$ and BA 9, $-38,19,30$ respectively). Neither source condition showed significant activations for the main effect of item retrieval or for the interaction between source and item retrieval. For the contrast of test minus study, there was the same pattern of activation in right middle frontal gyrus (BA 46) in both groups although the extent threshold was not exceeded. For the activity in hippocampal regions, both groups showed small effect sizes with large variances.

The main effects of source memory [(task $\mathrm{C}+$ task D) - $(\operatorname{task} A+\operatorname{task} B)]$ were observed in the right cerebellum, left middle frontal gyrus, medial aspect of the superior frontal gyrus, left supramarginal gyrus, and left inferior frontal gyrus (BA 45 and BA 47; Fig. 1a, Table 2). However, there were no significant activations observed for the main effect of item memory nor for the interaction between source and item memory. Simple effects of source memory were examined in two ways: (1) with no item retrieval (i.e. the contrast of task $C$, which required source judgments only on old items, with task A, the baseline task with old items; and (2) with item retrieval (i.e. the contrast of task $D$ requiring both source and item judgments, with task B, which required item judgments only). In the first contrast, only the superior frontal gyrus BA $9(2,44,36 ; Z \max =4.67$, $\mathrm{k}=490$ ) activation was significant; in the second contrast, the left inferior frontal gyrus BA $44(-38,14,34, \mathrm{Zmax}=4.18$, $\mathrm{k}=367$ ) was activated.

For the contrast of test minus study, there were significant activations observed in the following three regions: right supramarginal gyrus, right middle frontal gyrus, and left superior parietal lobule (Fig. 1b, Table 3). However, for the contrast of study minus test, there were no significant differences in activations.

Figure 2 shows the ROI analysis results for the main effects of item retrieval, source retrieval, their interaction, and the contrast between test and study in hippocampal regions. The only effect that was marginally significant was that activation was lower during test than study in the left anterior hippocampal region $(t(7)=2.03, p=0.08$, twotailed).

Event-related analyses: activation during the study phase conditionalized on correctness during the source test: If source retrieval was successful during the test phase of task $C$ (source retrieval alone), then we defined the corresponding item as correct source encoded during the study phase. If source retrieval was unsuccessful during the test phase of task $\mathrm{C}$, then we defined the corresponding item as an error due either to incorrect source encoded during the study phase or incorrect source retrieved during the test phase. Based on participants' performance (correct or error) on the source memory retrieval task (the test phase of task C), we compared the activation difference between correctly encoded and error trials during the study phase for this task using the event-related procedure.

Study phases of 32 runs (four runs for each participant) of task $C$ were included for this analysis. The mean accuracy for source retrieval during test was $78 \%$. The reaction times
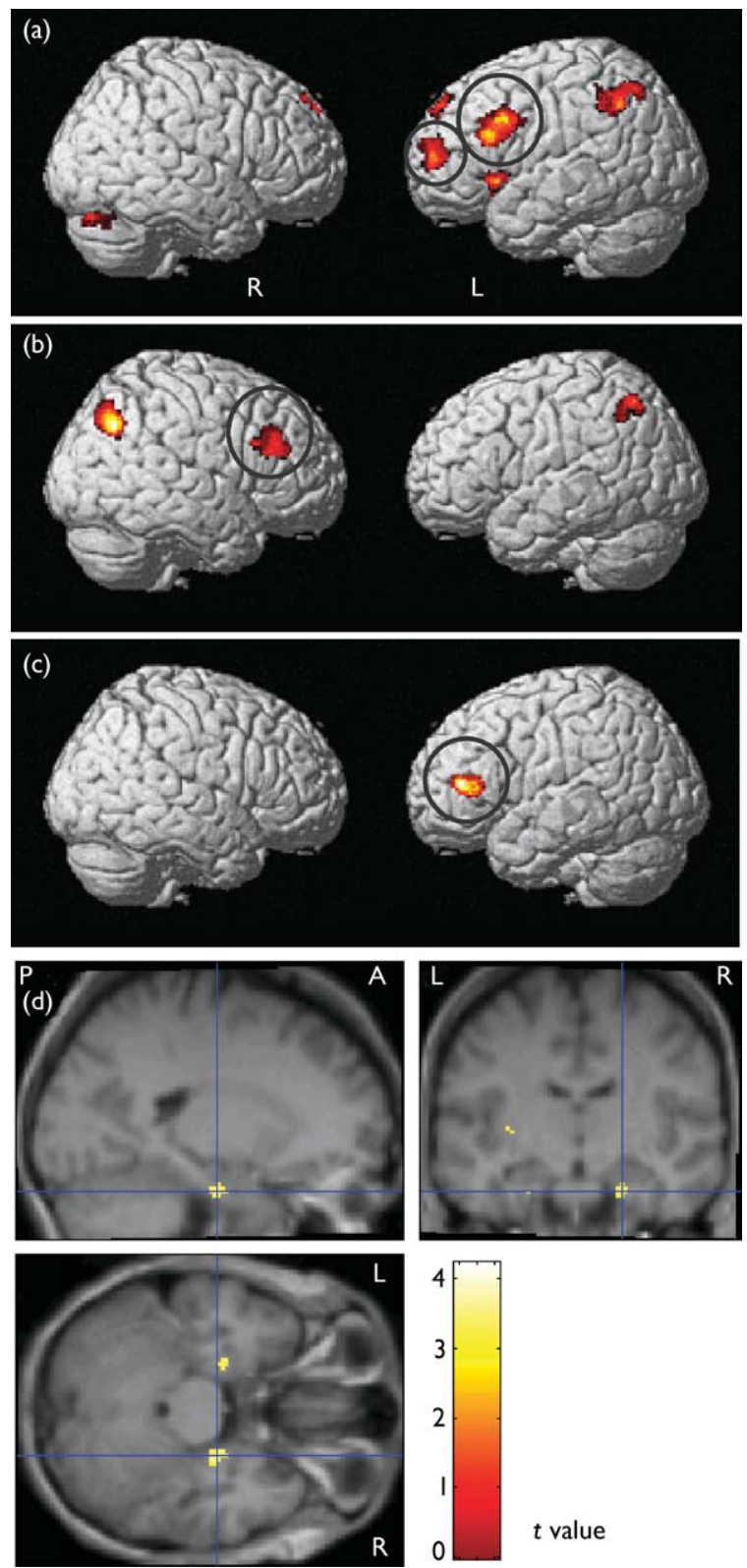

Fig. I. (a) Main effect of source memory. For item memory and interaction, there is no area which exceeded the extent threshold. (b) Contrasts of study and test tasks. Test $>$ study. For study $>$ test, there is no area which exceeded the extent threshold. Height threshold is $p<0.001$, and extent threshold is voxel $>216(p<0.001)$. (c) Difference of neural responses during study phase for correct and incorrect source retrieval trials at test phase. Height threshold is $p ;<0.00$ l (uncorrected), and extent threshold is voxel $>64$ ( $p<0.033$, uncorrected). Region: Inferior frontal gyrus (bordering middle frontal gyrus); Brodmann area: 45 ; voxel number $=188$, peak $Z$ value $=4.29$, Talairach coordinates: $-40,30,8$. (d) Difference of neural responses for correct and incorrect source retrieval trials during source retrieval. Height threshold is $p<0.00$ l (uncorrected), and extent threshold is voxel $\geq 8$. Regions: left parahippocampus; voxel number $=8$, peak $Z$ value $=3.35$, Talairach coordinates: $-24,-13,-21$; right parahippocampus; voxel number $=30$, peak $Z$ value $=3.64$, Talairach coordinates: $22,-15,-21$.

for correctly encoded and incorrectly encoded trials during study were not significantly different, $1301 \mathrm{~ms}$ vs $1309 \mathrm{~ms}$, $|t|<1$. However, there were differences in fMRI activa- 
Table 2. Brain regions showing significantly increased activity for the main effect of source memory. ${ }^{2}$

\begin{tabular}{|c|c|c|c|c|c|c|c|}
\hline \multirow[t]{2}{*}{ Region } & \multirow[t]{2}{*}{ Side } & \multirow[t]{2}{*}{ Brodmann area } & \multirow[t]{2}{*}{ Voxel number ${ }^{b}$} & \multirow[t]{2}{*}{ Peak Z } & \multicolumn{3}{|c|}{ Talairach coordinates } \\
\hline & & & & & $x$ & y & $\mathrm{z}$ \\
\hline Cerebellum (posterior lobe) & $\mathrm{R}$ & & 365 & 5.26 & 24 & -79 & -20 \\
\hline Middle frontal gyrus & $\mathrm{L}$ & 10 & 310 & 5.13 & -34 & 53 & 5 \\
\hline Superior frontal gyrus ${ }^{c}$ & & 9 & 682 & 4.92 & 2 & 44 & 36 \\
\hline Supramarginal gyrus & $\mathrm{L}$ & 40 & 489 & 4.58 & -32 & -50 & 38 \\
\hline Inferior frontal gyrus & L & 45 & 788 & 4.44 & -47 & 20 & 21 \\
\hline Inferior frontal gyrus & $\mathrm{L}$ & 47 & 247 & 4.05 & -44 & 19 & -4 \\
\hline
\end{tabular}

${ }^{a}(C+D)-(A+B)$.

${ }^{\mathrm{b} C l u s t e r-l e v e l} p_{\text {correct }}<0.005$.

'Medial aspect of the frontal gyrus.

Table 3. Brain regions showing significantly increased activity for the contrast of test and study phase. ${ }^{a}$

\begin{tabular}{|c|c|c|c|c|c|c|c|}
\hline \multirow[t]{2}{*}{ Region } & \multirow[t]{2}{*}{ Side } & \multirow[t]{2}{*}{ Brodmann area } & \multirow[t]{2}{*}{ Voxel number } & \multirow[t]{2}{*}{ Peak Z } & \multicolumn{3}{|c|}{ Talairach coordinates } \\
\hline & & & & & $x$ & y & z \\
\hline Supramarginal gyrus & $\mathrm{R}$ & 39 & 515 & 4.99 & 44 & -62 & 38 \\
\hline Middle frontal gyrus & $\mathrm{R}$ & 46 & 306 & 4.74 & 54 & 34 & 20 \\
\hline Superior parietal lobule & L & 7 & 270 & 4.73 & -30 & -59 & 49 \\
\hline
\end{tabular}

${ }^{a}$ The contrast is test minus study.
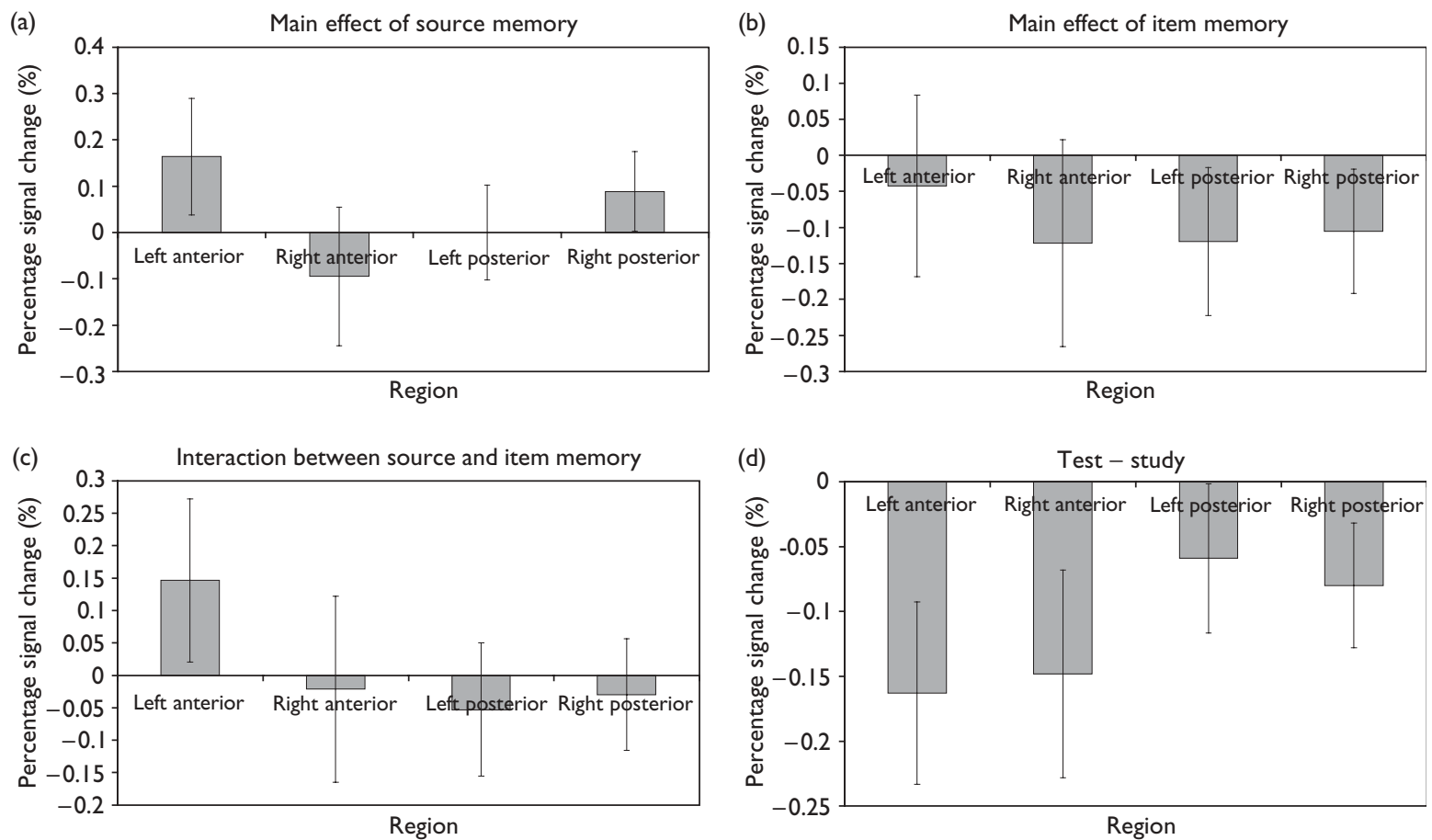

Fig. 2. Hippocampal ROI analysis results: main effects and interaction of four hippocampal regions to five comparisons. Error bars indicate s.e.m.

tions for correctly and incorrectly encoded trials during study. Figure 1c shows that the left inferior frontal gyrus (BA 45) had a significantly increased magnitude of activity for correct compared to incorrect source encoded trials during study.

Event-related analyses: activation during the test phase conditionalized on correctness during the source test: We also compared the activation difference between correct source retrieval and incorrect source retrieval trials during the source memory task (task C) using the event-related procedure. The mean reaction time of correct test trials (1339 ms) was significantly faster than that of incorrect test trials $(1788 \mathrm{~ms}, t(446)=6.58, p<0.01)$. Although no differences in prefrontal regions were observed, both left and right parahippocampal gyri showed an increased magnitude of activity for correct source test trials than for incorrect 
source test trials (Fig. 1d). This is consistent with the results of Schacter and Wagner [17].

\section{DISCUSSION}

Difference between source memory retrieval and item memory retrieval: In this experiment, we found increased activation in left prefrontal regions (BA 10, 45, 47) during source retrieval, suggesting that the left prefrontal regions are involved in source memory retrieval. Source memory retrieval tends to additionally recruit the left anterior prefrontal cortex near BA 10. We also found increased activation in the right cerebellum and left supramarginal gyrus. There were no significant activations observed for the main effect of item memory. Hippocampal regions showed no significant difference between source and item retrieval, nor between the retrieval test phase and the study phase.

The left prefrontal activations during source memory retrieval might be explained by three possibilities: (1) they reflect the involvement of effortful processes in attempted retrieval; (2) they are activated more by retrieval success, or (3) they reflect the involvement of subvocal speech as indicated by activations in Broca's area which may be helpful in retrieving more difficult information (note that this is a more elaborated version of possibility (1)). The event-related analysis on successful and unsuccessful source retrieval trials showed that only the degree of activation in bilateral parahippocampal regions, not in prefrontal regions, was correlated to the performance of source retrieval. Therefore, the second possibility, that prefrontal cortices are activated more by retrieval success, seems unlikely. On the other hand, the effort hypothesis cannot be ruled out because source memory retrieval was more difficult (as shown by both errors and reaction times) than item memory retrieval. The third explanation is also a possibility as the area activated (left BA 45 which is Broca's speech area) is the region involved in the organization of articulated language. However, the accompanying activations of BA 9, 10, and 47 are not well explained by the subvocalization hypothesis.

The effort hypothesis would also be consistent with the proposal that the prefrontal cortex is responsible for selecting, maintaining, updating, and rerouting information processing by a process of dynamic filtering [19]. From this perspective, the prefrontal cortex is not responsible for retrieving information from episodic memory, but rather for evaluating its significance in deciding which response to make. In particular, participants may execute elaborate decision-making strategies in deciding whether the strength of a memory trace is sufficiently strong to warrant a decision of old or the selection of a particular source, and the degree of involvement of prefrontal systems should parallel the complexity of these processes.

Activations during episodic memory encoding: We found that activation of left prefrontal cortex can predict memory performance. Consistent with a review of PET imaging studies on intentional episodic memory encoding [20], the present study showed that the left inferior frontal gyrus (BA 45) had greater activation for correctly encoded source. Our results are also consistent with a study showing that the ability to later remember a verbal experience is predicted by the magnitude of activation in left prefrontal and temporal regions during the learning experience [21]. However, it is not consistent with a study using complex color photographs which showed that the magnitudes of focal activation in right prefrontal cortex and in bilateral parahippocampal cortex predicted performance on a memory test [22].

Our results suggest a left frontal lateralization for the encoding of both source and item information. The left prefrontal cortex may be related to processes required to establish meaningful connections between an item and its source. Other possible explanations of the left PFC activation during successful encoding in the present study is that left prefrontal activation reflects an aspect of associative semantic processing [23], that verbal codes were used during encoding [24], or that the discrete nature of the sources such as color and voice assignments may be preferentially processed by left hemisphere systems.

Differences in activations between episodic memory encoding and retrieval: In this experiment, we found greater activation in right prefrontal regions (BA 46) during the test phases than during the study phases. These results suggest that the right prefrontal cortex plays a selective role in episodic memory retrieval. This is consistent with Tulving et al.'s HERA model [25] suggesting that right prefrontal regions deal more with retrieval than encoding of information from episodic memory. Because we found no significant hippocampal activation in the ROI analysis, our data are moot with respect to the HIPER model's predictions regarding the relative engagement of anterior and posterior hippocampal regions during encoding and retrieval operations [16]. It should be acknowledged, however that the small ROI used in this study may have decreased sensitivity to detect such effects.

\section{CONCLUSION}

The major purpose of this study was to investigate how brain activation differs when a participant attempts to retrieve source information compared to when the participant attempts to retrieve item information from episodic memory. We found that there is increased activation in left prefrontal areas for source retrieval, but not for item retrieval. However, the results of hippocampal activations were not significantly different between source and item retrieval. Our results suggest a unitary view of source and item memory such that source memory involves more processes in attempted encoding and retrieval of source information in addition to the encoding and retrieval of item information itself, thereby supporting the single process over the dual process model of item and source retrieval.

\section{REFERENCES}

1. Tulving E. In: Tulving E and Donaldson W, (eds). Organization of Memory. New York: Academic Press; 1972, pp. 381-403.

2. Johnson MK, Hashtroudi S and Lindsay DS. Psychol Bull 114, 3-28 (1993).

3. Moscovitch M and Winocur G. In: Craik FIM and Salthouse TA (eds). The Handbook of Aging and Cognition. Hillsdale, NJ: Erlbaum, 1992, pp. 315-372. 
4. Shimamura AP, Janowsky JS and Squire LR. Neuropsychologia $\mathbf{2 8}, 803-813$ (1990).

5. Janowsky JS, Shimamura AP and Squire LR. Neuropsychologia 27, 10431056 (1989).

6. Schacter DL. Psychobiology 15, 21-36 (1987).

7. Squire LR. In: Schacter DL and Tulving E (eds). Memory Systems. Cambridge, MA: MIT Press; 1994, pp. 203-231.

8. Glisky EL, Polster MR and Routhieaux BC. Neuropsychology 9, 229-235 (1995).

9. Cabeza R, Mangels J, Nyberg L et al. Neuron 19, 863-870 (1997).

10. Squire LR and Zola-Morgan S. Science 253, 1380-1386 (1991).

11. Henkel LA, Johnson MK and De Leonardis DM. J Exp Psychol Gen 127, 251-268 (1998).

12. Nolde SF, Johnson MK and D'Esposito M. Neuroreport 9, 3509-3514 (1998).

13. Buckner RL, Koutstaal W, Schacter DL et al. Neuroimage 7, 151-162 (1998).
14. Cycowicz YM, Friedman D, Rothstein M and Snodgrass JG. Journal of Experimental Child Psychology 65, 171-237 (1997).

15. Friston KJ, Price CJ, Fletcher P et al. Neuroimage 4, 97-104 (1996).

16. Lepage M, Habib R and Tulving E. Hippocampus 8, 313-322 (1998).

17. Schacter DL and Wagner AD. Hippocampus 9, 7-24 (1999).

18. Strange BA, Fletcher PC, Henson RNA et al. Proc Natl Acad Sci USA 96, 4034-4039 (1999).

19. Shimamura AP. Psychobiology 28, 207-218 (2000).

20. Cabeza R and Nyberg L. J Cogn Neurosci 9, 1-26 (1997).

21. Wagner AD, Schacter DL, Rotte M et al. Science 281, 1188-1191 (1998).

22. Brewer JB, Zhao Z, Desmond JE et al. Science 281, 1185-1187 (1998).

23. Dolan RJ and Fletcher PC. Nature Neurosci 388, 249-256 (1997).

24. Kelley WM, Miezin FM, McDermott KB et al. Neuron 20, 927-936 (1998).

25. Tulving E, Kapur S, Craik FIM et al. Proc Natl Acad Sci USA 91, 2016-2020 (1994).

Acknowledgements: We are very grateful for the help we obtained from Dr Joseph A. Helpern, Dr Craig A. Branch, Mr Raj Sangoi, Mr Joseph Grun, DrTodd Lencz, and Dr Houwei Wu. Our further thanks are directed to Dr Michael I. Posner, Dr Edward E. Smith, and Dr Wendy Suzuki for their very helpful advice on the experiment. 